\title{
VALUTAZIONE DELTEST MONOLISA HCV AG-AB ULTRA IN UN LABORATORIO DI SIEROIMMUNOLOGIA: DATI PRELIMINARI
}

\author{
Colao M.G., Parri F. \\ Laboratorio di Sieroimmunologia, \\ Azienda Ospedaliera Universitaria Careggi, Firenze
}

\section{Introduzione}

Monolisa HCV Ag-Ab Ultra è un dosaggio immunoenzimatico che consente la determinazione simultanea degli anticorpi e dell'antigene del capside di HCV.

Scopo dello studio è confrontare i risultati preliminari di questo test con quelli del metodo in uso per lo screening degli anticorpi anti HCV (Cobas Core anti HCV EIA II, Roche), e valutare la sua applicabilità alla routine del nostro Laboratorio.

\section{Materiali e Metodi}

Prodotti utilizzati

- Monolisa HCV Ag-Ab Ultra, Bio-Rad.

- Cobas Core anti HCV EIA II, Roche

- Test di conferma RIBA HCV 3.0, Chiron.

Campioni

- 644 sieri della routine, conservati a $+4^{\circ}$, eseguiti nell'arco di 4 giorni.

- 107 sieri selezionati nell'arco di 2 mesi perché debolmente reattivi o dubbi al test di screening, congelati a $-80^{\circ}$.

Tutti i campioni positivi, dubbi e/o discordanti sono stati analizzati ulteriormente con il test RIBA HCV 3.0.

\section{Risultati}

Dei 644 sieri della routine: 598 sono risultati negativi e 38 positivi con entrambi i metodi, confermati dal test RIBA; 8 campioni sono risultati discordanti.

Dei 107 sieri selezionati: 46 sono stati confermati dal test Ag-Ab; 61 campioni sono risultati discordanti.

I campioni discordanti hanno RIBA indeterminato o negativo. Conclusioni

Sui 644 sieri della routine il test Monolisa HCV Ag-Ab ha dimostrato una concordanza con il test Cobas anti HCV del 98.8\%. 3 campioni sono risultati debolmente reattivi con il test $\mathrm{Ag}-\mathrm{Ab}$ e negativi con Cobas anti HCV; il RIBA ha dato esito negativo, ma non è stato possibile eseguire test di biologia molecolare per HCV RNA.

Sui 107 sieri selezionati la concordanza tra i test Monolisa HCV Ag-Ab e Cobas anti HCV è del $43 \%$.

I campioni discordanti (screening debolmente reattivo e test $\mathrm{Ag}-\mathrm{Ab}$ negativo) sono risultati per il $65.1 \%$ RIBA negativo e per il $34.9 \%$ RIBA indeterminato. Nessun campione è risultato RIBA positivo.

I vantaggi che ci sembra poter identificare nell'uso routinario del test $\mathrm{Ag}-\mathrm{Ab}$ riguardano la migliore concordanza con il RIBA (eliminazione di falsi positivi allo screening) e la riduzione della fase finestra, grazie alla possibilità di evidenziare l'antigene del capside di HCV. 